\title{
Klinisches Bild und Diagnostik des angeborenen portosystemischen Shunts beim Hund
}

\author{
Ingmar Kiefer, Beate Bosch, Claudia Köhler, Franziska Eberhardt, Eberhard Ludewig
}

Als kleines Dankeschön für ein perfektes Teamplay Frau Professor Alef gewidmet.

Im Gegensatz zum Menschen, bei dem ein erworbener portosystemischer Shunt meist nur sekundär durch Erkrankungen der Leber auftritt, kommt der portosystemische Shunt beim Hund öfter angeboren als erworben vor. Die Häufigkeit der Erkrankung wird kritisch diskutiert. Man geht jedoch von einer erheblichen Dunkelziffer aus, insbesondere weil die Symptome mannigfaltig sind und die Ausprägung sehr unterschiedlich ist. Die Erkrankung zählt zu den beim Hund beschriebenen Erbkrankheiten.

Beschrieben wurde der portosystemische Shunt (PSS) als eine vaskuläre Anomalie beim Hund erstmals 1949. Aufgrund des vermehrten Auftretens der Erkrankung bei bestimmten Rassen und sogar Familien geht man von einer Erbkrankheit aus. Dieser Annahme entspricht auch die Definition [22], wenngleich der Erbgang noch nicht 100\%ig nachgewiesen wurde. Es wird von einer komplexen autosomalen Vererbung ausgegangen [15]. So wird in einzelnen Studien $[26,27]$ von einer 36-fach erhöhten Wahrscheinlichkeit bei Yorkshire Terriern ausgegangen. Einzelne Zuchtversuche mit 2 Elterntieren, die an einem PSS erkrankt waren, ergaben jedoch nur gesunde Nachkommen [26]. Die Studie [26] wurde allerdings nicht mit letzter Konsequenz weitergeführt, weshalb die Aussagekraft kritisch bewertet werden sollte. Ähnliche Aussagen gibt es zur Prävalenz beim Irischen Wolfshund [23, 24, 28], weshalb in einigen Ländern der Zuchtverband bei diesen Hunden die Kontrolle der Ammoniakwerte vor der Zuchtzulassung im Plasma empfiehlt [18]. Neben den schon genannten Rassen tritt die Erkrankung unter anderen auch bei Cairn Terriern [29], Norfolk Terriern, Maltesern und Australian Cattle Dogs gehäuft auf [25], nach Meinung der Autoren auch bei Retrievern, Hovawarts, Rauhaardackeln, West Highland White Terriern und Zwergschnauzern. Auch bei Mischlingen tritt die Erkrankung auf, wenngleich scheinbar deutlich seltener als bei Rassehunden [17]. Eine Geschlechtsdisposition scheint nicht vorzuliegen [19].

\section{Einteilung}

Es gibt verschiedene Formen des PSS, wobei sich eine einfache Unterteilung in intra- und extrahepatische Formen für das Besitzergespräch bewährt hat. Kleine Hunderassen erkranken eher an einem extrahepatischen Shunt, große Rassen, wie zum Beispiel der Irische Wolfshund, an einem intrahepatischen Shunt. Generell wird von einem Verhältnis von ca. 2:1 zwischen extra- und intrahepatischem Shunt ausgegangen [5, 17].

\section{Klinisches Bild}

Nur wenige klar definierte Erkrankungen können sich so unterschiedlich präsentieren wie der PSS. Es gibt Tiere, die über viele Jahre weitgehend symptomlos sind und dann nur zufällig durch eine lange Nachschlafphase nach einer Zahnsanierung auffallen, aber auch Patienten, die sich direkt nach der Futterumstellung als Welpe mit typischem Drangwandern präsentieren. Gerade diese Vielfalt und Varianz der Symptome macht es aus der Sicht der Autoren nicht immer leicht, an einen PSS zu denken.

In den letzten Jahren scheint sich das Erkrankungsbild geändert zu haben, was aber auch an der erhöhten Sensibilität der Tierärzte für diese Erkrankung liegen mag. So ist der subjektive Eindruck der
Rasseprädisposition -

portosystemischer Shunt

- Australian Cattle Dog [25]

- Cairn Terrier [29]

- Hovawart [15]

- Irischer Wolfshund [23, 24, 28]

- Malteser [25]

- Norfolk Terrier (eigene Daten)

- Rauhaardackel [15]

- Retriever [15]

- West Highland White Terrier (eigene Daten)

- Yorkshire Terrier [15, 26]

- Zwergschnauzer [15]

Autoren, dass vor einigen Jahren die neurologischen Symptome (Drangwandern nach der Futteraufnahme, Kopf gegen die Wand drücken) am häufigsten vorkamen, jetzt aber unspezifische Symptome wie eine erhöhte Wasseraufnahme und erhöhter Urinabsatz auffälliger sind.

Vereinfacht kann man die Symptome in 3 Gruppen einteilen:

- zentralnervös

- gastrointestinal

- urologisch

\section{Zentralnervöse Symptome}

Die Auffälligkeiten des ZNS reichen von Lethargie, episodischem Auftreten einer Belastungsintoleranz, Ataxie, Kopf an die Wand pressen, Orientierungslosigkeit, temporärer Blindheit, Verhaltensänderung, Allotriophagie, extremer Salivation, Drangwandern, Anfällen bis hin zum Koma [14, 15, 24, 25].

Für das Auftreten der Symptome, die häufig auch als hepatoenzephales Syndrom bezeichnet werden, ist wohl maßgeblich der erhöhte Gehalt von Ammoniak im Plasma verantwortlich. Dieser entsteht zum großen Teil durch den bak- 
teriellen Eiweißabbau im Kolon. Physiologischerweise wird das Ammoniak in der Leber nahezu vollständig aus dem Blut entfernt und zu Harnstoff umgebaut. Durch die Umgehung der Leber infolge des PSS gelangt das Ammoniak stattdessen in den großen Blutkreislauf und somit auch ins Gehirn. Ammoniak ist exzitotoxisch und mit einer erhöhten Freisetzung von Glutamat (hauptexzitatorischer Transmitter im Gehirn), und somit mit einer Überaktivierung von Glutamatrezeptoren, assoziiert. Desweiteren sind mehr als 20 verschiedene Substanzen, wie z.B. Tryptophan, Glutamin, Gammaaminobuttersäure, aromatische Aminosäuren, kurzkettige Fettsäuren bei Patienten mit PSS im Blut erhöht. Diese können die neuronale und Astrozytenfunktion beeinflussen. Vollständig ist der Wirkungsmechanismus aber noch nicht geklärt [14].

\section{Gastrointestinale Symptome}

$\mathrm{Zu}$ den gastrointestinalen Symptomen muss man neben Erbrechen und Durchfall [7, 14] auch das Zurückbleiben von Wachstum [15] und Entwicklung dazuzählen.

Ursächlich für die verzögerte Entwicklung der Hunde ist die massiv reduzierte Durchblutung der Leber. Das Organ erhält physiologisch ca. 70\% des Blutflusses und $50 \%$ des Sauerstoffs aus der Pfort-

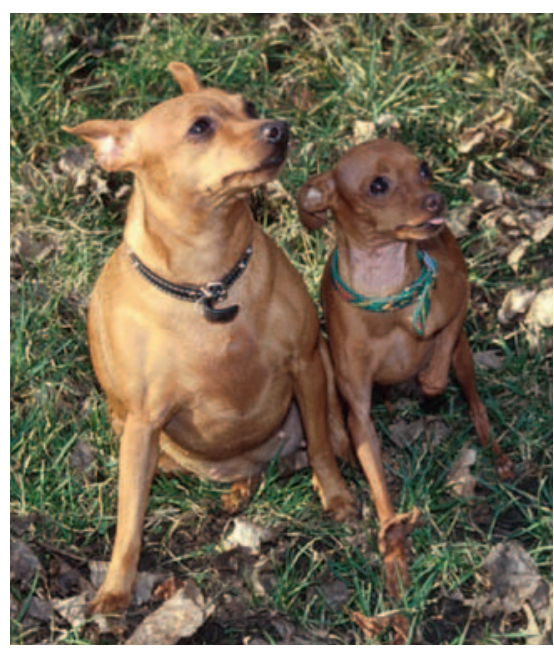

Abb. 1 Größenvergleich zweier Wurfgeschwister: Deutlich sichtbar der enorme Unterschied zwischen dem an einem portocavalen Shunt erkrankten rechten Tier und dem gleichaltrigen Geschwistertier. Der Größenunterschied war 8 Monate nach dem erfolgreichen Verschluss nicht mehr auffällig. ader. Durch das Vorliegen eines PSS reduziert sich der Gesamtblutfluss der Leber, je nach Ausprägung des Shunts, auf weniger als 50\% [7, 14]. Dies führt nicht nur zu einer Einschränkung der Entgiftungsfunktion, sondern auch dazu, dass die Leber sich nicht adäquat entwickelt. Als Konsequenz sind die Hunde häufig kleiner als die nicht erkrankten Wurfgeschwister und behalten mitunter auch ihr „Welpenfell“ [1] ( $\triangleright$ Abb. 1). Diese Entwicklungsstörung ist somit indirekt als Symptom des GIT zu werten.

Mitunter lehnen die betroffenen Tiere das klassische Hundefutter ab - ob dies nur zufällig geschieht oder einen Schutzmechanismus darstellt, bleibt fraglich. Sekundär können weitere gastrointestinale Symptome durch die häufig recht stark ausgeprägte Allotriophagie auftreten. Auch Ulzera im Gastrointestinaltrakt können auftreten, spielen klinisch jedoch eher selten eine Rolle.

\section{Urologische Symptome}

Relativ häufig zeigen Tiere mit einem PSS eine ausgeprägte Polyurie/Polydipsie (PU/PD) [14, 15].

Ursächlich für die primär auftretende PU ist das Fehlen des Harnstoffgradienten im Tubulussystem. Dieser Gradient ist maßgeblich für die Rückresorption des Primärharns verantwortlich. Fehlt der Harnstoff, erfolgt eine mangelhafte Konzentration des Urins, was dann als Ausgleich sekundär zu einer vermehrten Wasseraufnahme führt. Bedingt durch den erhöhten Ammoniakspiegel kommt es zum Ausfallen von Ammoniumuraten, die sich häufig sonografisch als diffuse Verkalkungen in den Nieren oder als Harngrieß in der Blase zeigen können. Je nach Ausprägung kann es aber auch zu soliden Nierenbecken- und Harnblasensteinen kommen, die in der Regel in der Projektionsradiografie nicht darstellbar sind. Sekundär führen diese zu Entzündungen bis hin zur Hämaturie.

\section{Labordiagnostik}

Es gibt zahlreiche Veränderungen, die labordiagnostisch auf das Vorliegen eines PSS hindeuten. Vorab muss jedoch darauf hingewiesen werden, dass kein Befund einen Shunt sicher ausschließt, was den Umkehrschluss zulässt, dass im Zwei- felsfall immer eine eindeutige Bildgebung erforderlich ist.

Typischerweise zeigen Tiere mit einem PSS

- einen Harnstoffwert deutlich unterhalb des Referenzbereichs und

- einen Ammoniakwert deutlich oberhalb des Referenzbereichs (meist deutlich über $120 \mu \mathrm{mol} / \mathrm{l})[14,15]$.

Doch auch wenn der Ammoniakwert normal ist, kann ein PSS vorliegen [13, 14]. Ebenso ist es nicht möglich, eine Korrelation zwischen dem Ammoniakwert und den klinischen Symptomen herzustellen.

\section{Gallensäurenstimulationstest}

Der Gallensäurenstimulationstest hat sich in der Shuntdiagnostik etabliert. Der Test überprüft die Leberfunktion, wobei der Ablauf je nach Schule unterschiedlich ist. Am einfachsten erscheint die Durchführung unter der Anwendung eines Cholezystokinetikums:

- Bestimmung des Gallensäurenbasalwerts

- anschließende Gabe des Cholezystokinetikums Ceruletid

- nach 30 min: Bestimmung des Gallensäurenstimulationswerts

Da Ceruletid nur noch schwierig zu beschaffen ist, kann die Entleerung der Gallenblase alternativ durch eine fettreiche Reizmahlzeit (zum Beispiel a/d-Diät, evtl. zusätzlich mit einem Ei vermengt, andere Autoren nutzen kommerzielle Leberdiäten) ausgelöst werden [6]. Auch die Stimulation mit einer proteinreichen Diät kann in Einzelfällen zu Problemen führen. Da der Patient auf die Reizmahlzeit deutlich langsamer als auf die Injektion reagiert, erfolgt die Bestimmung der stimulierten Werte je nach Literaturstelle $[6,15,11]$ erst nach 90-120 Minuten. Die Referenzwerte differieren je nach Labor, als physiologisch gelten üblicherweise:

- Basalwerte bis $20 \mathrm{mmol} / \mathrm{l}$

- Stimulationswerte bis $40 \mathrm{mmol} / \mathrm{l}$

Beim Shuntpatienten können die Stimulationswerte weit über $100 \mathrm{mmol} / \mathrm{l}[14$, 15] liegen. Erhöhte Werte weisen auf eine eingeschränkte Leberfunktion hin. Beim jungen Hund ist dieses häufig Folge eines 
PSS, kann aber auch andere Ursachen (z.B. Leberfibrose, Zirrhose, chronische Hepatitis) haben. In einer australischen Studie aus dem Jahr 1995 wurden erhöhte Gallensäuren bei Maltesern beschrieben, die Ursache ist jedoch unbekannt. Bei einem Teil der Tiere wurden Leberbiopsien durchgeführt, jedoch keine Portografie. Da auch Malteser zu den häufig betroffenen Rassen gehören, kann weder ein PSS noch eine mikrovaskuläre Dysplasie sicher ausgeschlossen werden.

\section{Ammoniaktoleranztest}

Der früher häufiger durchgeführte Ammoniaktoleranztest [21] (Applikation einer definierten Menge Ammoniak) wird zurzeit als sehr problematisch angesehen, da dieser beim Shuntpatienten zu erheblichen neurologischen Ausfällen, im schlimmsten Fall auch zum Tode, führen kann. Dies gilt auch für Patienten, die weitgehend klinisch unauffällig sind.

\section{Weitere Parameter}

Neben diesen vergleichsweise spezifischen Parametern zeigt sich die eingeschränkte Leberfunktion in einer Vielzahl von veränderten Werten, die alle einzeln nicht spezifisch sind. In Kombination erhärten sie jedoch die Verdachtsdiagnose. Dies zeigt sich zum Beispiel in einem oft reduzierten Serumgesamteiweiß, der sich sowohl aus einem er- niedrigten Albumingehalt als auch durch eine erniedrigte Gammaglobulinfraktion ergibt.

Im Gegensatz dazu sind die Werte von Aspartat-Aminotransferase (AST), Alanin-Aminotransferase (ALT) und Bilirubin meist im Referenzbereich. In der Literatur [15] wird die alkalische Phosphatase (AP) beim Patienten mit PSS mitunter als deutlich erhöht beschrieben. Im Patientengut der Autoren waren die Werte meist im Referenzbereich bzw. nur marginal erhöht.

Der Cholesterinwert ist bei den Shuntpatienten häufig erniedrigt. Zusätzlich zeigen die Tiere mitunter eine moderate Hypoglykämie. Hämatologisch sind eine milde Anämie und eine Mikrozytose nachweisbar.

Aufgrund der Fehlfunktion der Leber haben fast alle Shuntpatienten labordiagnostisch nachweisbare Gerinnungsstörungen. Erstaunlicherweise spielen diese klinisch - auch bei den chirurgischen Eingriffen - keine Rolle.

Wertvoll kann der Nachweis von Ammoniumuraten im Urin sein, der Nichtnachweis schließt aber den PSS nicht aus.

\section{Bildgebende Diagnostik Radiografie}

Vergleichbar mit der Labordiagnostik ist der Nachweis des PSS in der Projektions- radiografie nicht möglich, es können jedoch weitere Hinweise gewonnen werden. So hat ein Großteil der Hunde eine sehr kleine Leber [14, 15], sodass der Magen weit in den rippengestützten Anteil der Bauchhöhle verlagert ist ( $\triangleright$ Abb.2). Auch zeigen die Tiere häufig eine Renomegalie. Die schon beschriebenen Blasen- oder Nierensteine lassen sich in der Regel in der Projektionsradiografie nicht nachweisen. In seltenen Fällen sind sie durch eine Kalziumverschmutzung sichtbar. Oft findet man auch Fremdkörper in dem Magen-DarmTrakt als Zeichen der Allotriophagie.

\section{Sonografie}

Etwas sensitiver ist die sonografische Diagnostik [9, 10, 16]. Auch hier muss zwischen dem eindeutigen Nachweis des Shuntgefäßes und den sekundären Hinweisen unterschieden werden. In einer retrospektiven Auswertung des eigenen Patientenguts haben die Autoren sonografisch $100 \%$ (Goldstandard war die jejunale Portografie) der intrahepatischen Shunts ( Abb.3, $\triangleright$ Abb.4) und 76\% der extrahepatischen Shunts eindeutig diagnostiziert. Diese Werte decken sich auch mit den in der Literatur gemachten Angaben [20].

Sekundäre Hinweise wie das Fehlen der Portalverzweigung, die kleine Leber, Turbulenzen in der V. cava, die Vergröße-

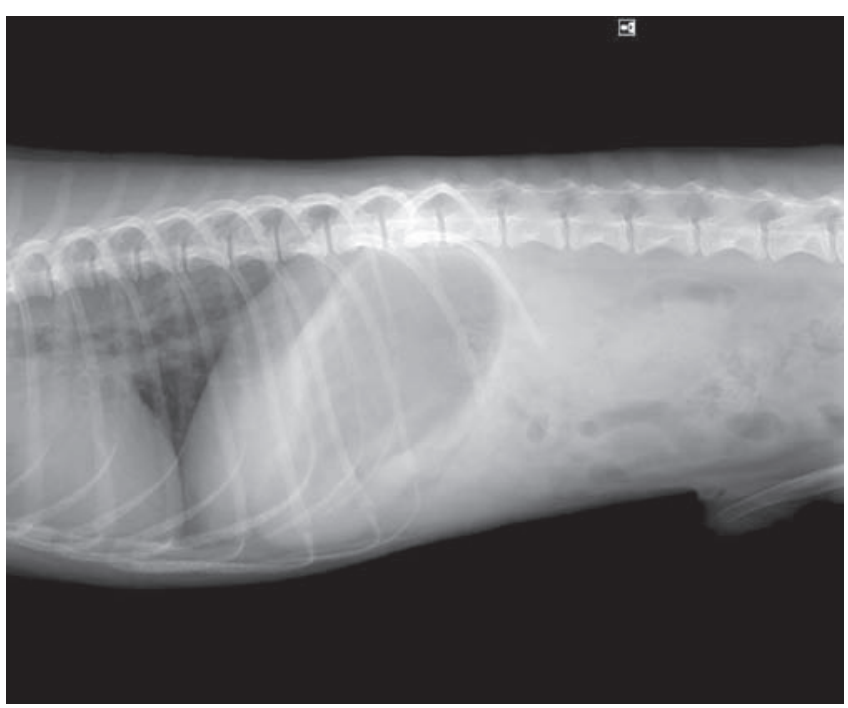

Abb. 2 Laterolaterales Röntgenbild eines 8 Monate alten Dackels. Durch den gasgefüllten Magen wird die extrem kleine Leber noch deutlicher. Die Renomegalie kann in diesem Bild nur erahnt werden.

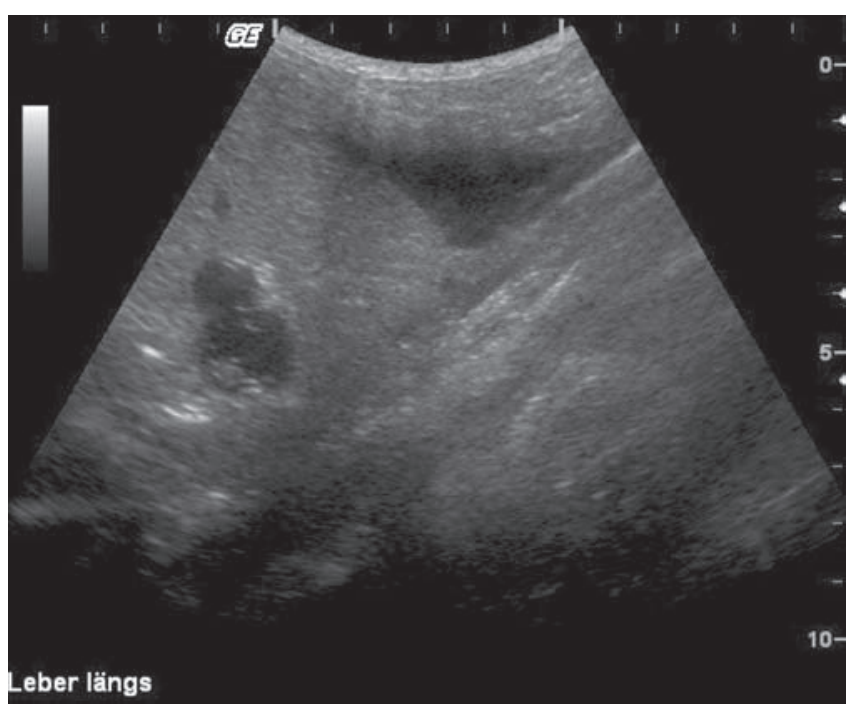

Abb. 3 Sonografie-Darstellung der Leber eines 6 Monate alten Hovawarts. Das Tier wird in Rücklage untersucht, ventral stellt sich ein mgr. Aszites dar. Die Leber zeigt sich vermehrt reflexreich und mit nur wenigen Gefäßen. Die beiden kreisrunden Strukturen stellen den intrahepatischen Shunt dar. 
rung der Nieren und der Milz, Nachweise von Kristallen im Nierenparenchym und von Kristallen in der Harnblase sind als weitere Verdachtsmomente zu werten und sollten den Untersucher kritisch über eine weitere Bildgebung nachdenken lassen.

Eine Besonderheit stellen hier die Harnkristalle dar. Die meisten Kristalle in der Harnblase zeigen sonografisch ein ausgeprägtes Sedimentationsverhalten. Am- moniumurate hingegen sedimentieren nur sehr langsam und zeigen ein Art „Schwebeverhalten“, wodurch sie meist sonografisch relativ sicher von anderen Kristallarten unterschieden werden können ( $\triangleright$ Abb. 5).

Zusammenfassend gilt für die Sonografie, dass der eindeutige Nachweis des Shunts zwar beweisend, der negative Befund jedoch nicht ausschließend ist.

\section{.konkret \\ Ammoniumurate lassen sich durch ihr "Schwebeverhalten“ sonografisch relativ sicher von anderen Kristallarten unterscheiden.}

\section{Szintigrafie}

Die lange Zeit im angloamerikanischen Bereich propagierte rektale Szintigrafie

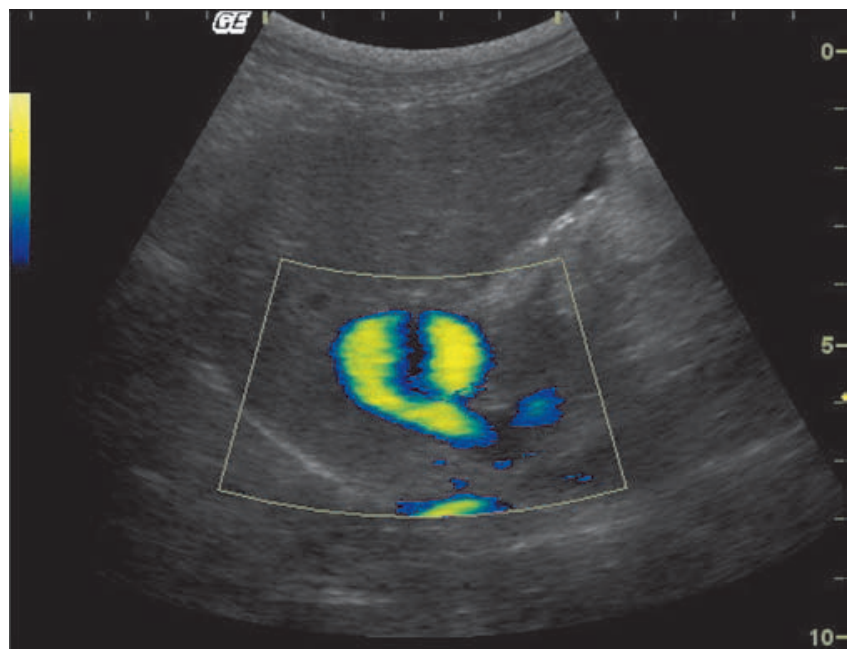

Abb. 4 Darstellung eines intrahepatischen Shunts mit dem amplitudenkodierten Doppler. Die Darstellung des intrahepatischen Shunts gelingt meist schon im B-Bild, der Doppler zeigt jedoch den Blutfluss besser an. Dabei spielt es keine Rolle, ob mit dem klassischen Farbdoppler oder dem amplitudenkodierten Doppler gearbeitet wird.

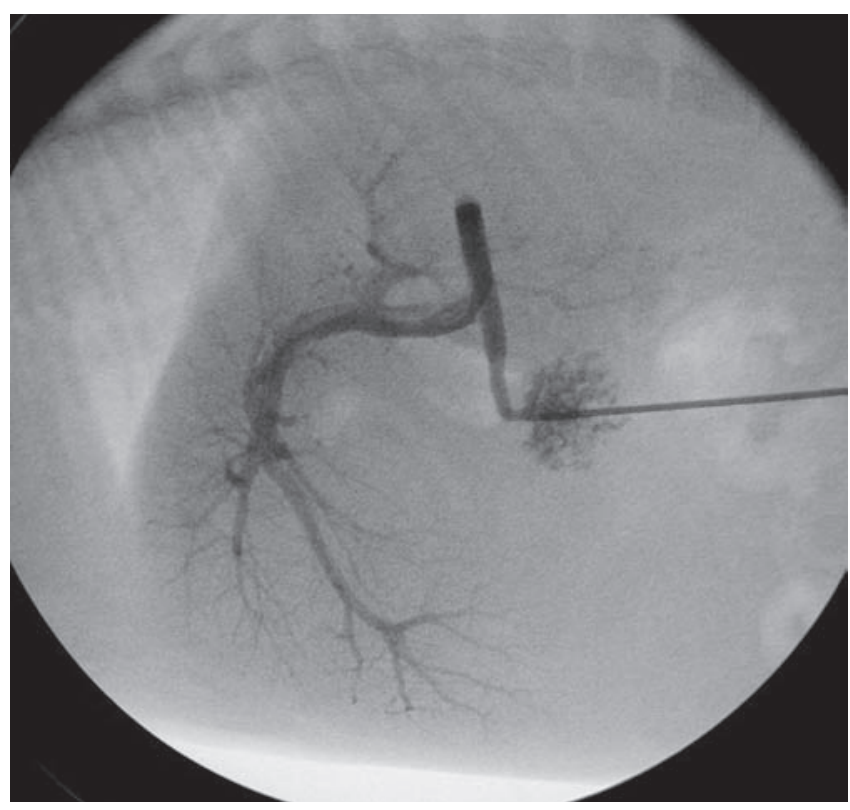

Abb. 6 Physiologisches Portogramm im laterolateralen Strahlengang. Die Nadel wurde perkutan unter sonografischer Kontrolle in die Milz platziert und dann unter Durchleuchtung das Kontrastmittel appliziert. Die Verzweigung der Portalgefäße ist sehr gut erkenntlich.

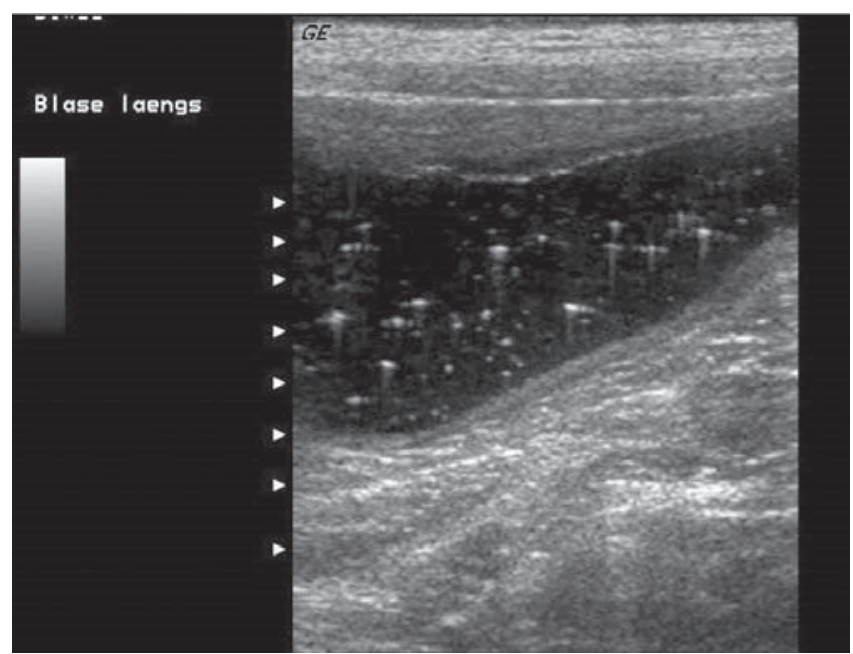

Abb. 5 In der Blase dieses Dackels stellt sich der Ammoniumuratgrieß in seiner typischen Art dar. Der Grieß zeigt eine Tendenz zur Flotation und das typische "Glitzern“.

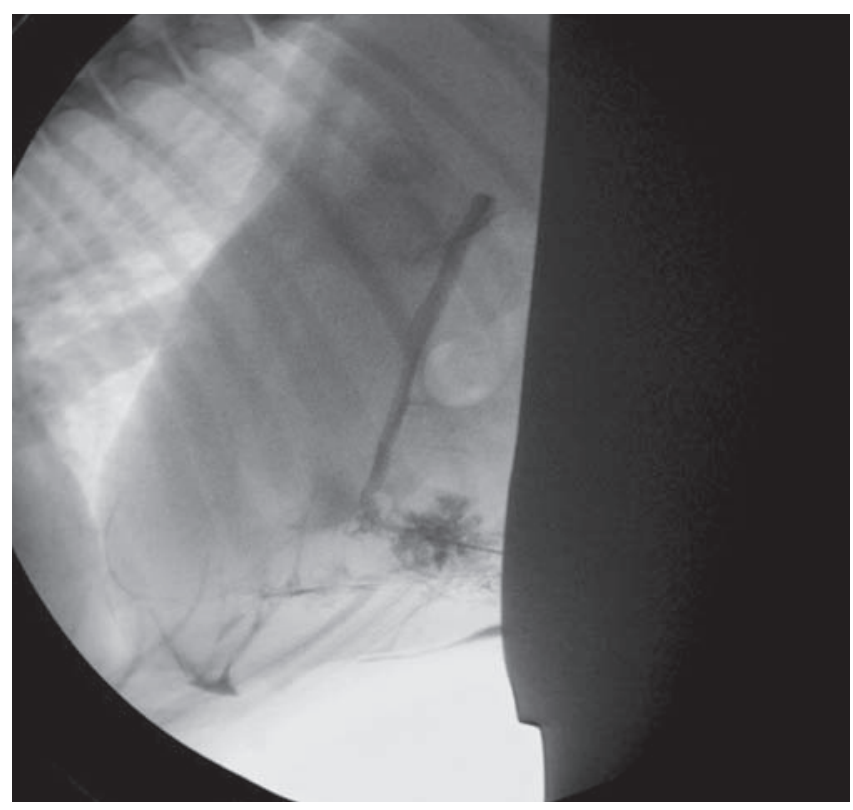

Abb. 7 Bei dieser Splenoportografie fließt das Kontrastmittel direkt über einen portocavalen Shunt in die V. cava caudalis ab, in der Leber nehmen keine Gefäße Kontrastmittel auf. Ein geringer Teil des Kontrastmittels ist in die freie Bauchhöhle ausgetreten. 
erscheint den Autoren nicht mehr zeitgemäß. Bei diesem Verfahren wird rektal ein Radionuklid verabreicht [8]. Dieses wird über die Darmmukosa resorbiert und sollte sich physiologisch zunächst in der Leber und dann erst im Herzen anreichern. Beim Vorliegen eines PSS reichert sich das Nuklid gar nicht oder deutlich schwächer in der Leber an [2]. Das Verfahren erscheint den Autoren jedoch relativ aufwendig und die Aussagekraft aufgrund der schlechten örtlichen Auflösung unzureichend. Gleiches gilt auch für die Szintigrafie nach Injektion in die Milz.

\section{Splenoportografie}

Als Alternative zur jejunalen Portografie hat sich die sogenannte Splenoportografie etabliert. Das Verfahren wurde zunächst sehr kritisch bewertet, weil es häufig nach der Blindpunktion der Milz zu erheblichen Blutungen gekommen ist. Um dies zu vermeiden, wurde dann für eine Splenoportografie (wie bei einer jejunalen Portografie) das Abdomen eröffnet und das Kontrastmittel unter Sichtkontrolle in die Milz appliziert. Da der Aufwand jedoch nahezu identisch zur jejunalen Portografie ist, erschien auch diese Modifikation wenig sinnvoll. Erst mit dem Einzug der Sonografie in die Kleintiermedizin hat sich das Verfahren als echte Alternative behauptet.

Mithilfe der Sonografie wird die Injektionsnadel sicher in der Milz platziert, bevor das nicht ionische, wasserlösliche jodhaltige Kontrastmittel (zum Beispiel Imeron ${ }^{\circledR} 300$ oder Solutrast ${ }^{\circledR} 250$ ) in die Milz unter Durchleuchtungskontrolle injiziert wird. Der Weg des Kontrastmittels wird über die V. lienalis zur V. portae und je nach Verlauf genau wie bei der jejunalen Portografie verfolgt und aufgezeichnet ( $\triangleright$ Abb.6, $\triangleright$ Abb.7). Mittels Subtraktionsangiografie (bei der nur das fließende Kontrastmittel dargestellt wird) kann der Verlauf noch deutlicher sichtbar gemacht werden, da die Darstellung der Umgebung (Leber, Darm usw.) unterdrückt wird. In einer Vergleichsstudie zur jejunalen Portografie mit 43 Patienten hat sich das Verfahren als sehr genau erwiesen. Lediglich bei einem Patienten gab es einen Unterschied in der Aussage, der durch einen zu klein gewählten Durchleuchtungsausschnitt verursacht wurde (eigene Daten). Die für die Diagnostik benötigte Narkose ist in der Regel sehr kurz (der Vorgang dauert weniger als 10 Minuten) und kann z.B. mit Propofol erhalten werden.

Der Diagnostiker sollte trotzdem kritisch überlegen, bei welchem Patienten er diese Methode anwendet. Die Splenopor- tografie erscheint bei Patienten, die relativ sicher einen PSS haben, wenig sinnvoll, da für die anschließende Therapie in der Regel das Abdomen eröffnet werden muss (Ausnahme Coilimplantation). In diesen Fällen kann daher direkt eine jejunale Portografie durchgeführt werden. Bei Patienten, die höchstwahrscheinlich keinen PSS aufweisen, erscheint den Autoren die Splenoportografie sehr zweckmäßig, da sie schnell und einfach durchzuführen ist.

\section{Computertomografie}

Mit dem Einzug von schnellen mehrzeiligen Computertomografen (CT) hat sich eine weitere Alternative zur Diagnostik des PSS ergeben [4, 12]. Hierbei gibt es mehrere Möglichkeiten, die Untersuchung durchzuführen, was nicht unerheblich von der Ausstattung des Tomografen abhängt. Die Untersuchung sollte gestartet werden, wenn das Kontrastmittel nach der periphervenösen Applikation am stärksten in der Portalvene anflutet $(\triangleright$ Abb. 8, $\triangleright$ Abb.9). Die einfachste, aber auch ungenaueste Methode ist die Schätzung des richtigen Untersuchungszeitpunkts anhand von Erfahrungswerten. Da die portalvenöse Phase im Gegensatz zur arteriellen Phase vergleichsweise lang ist [3], funktioniert das Verfahren zwar, bringt aber nicht immer

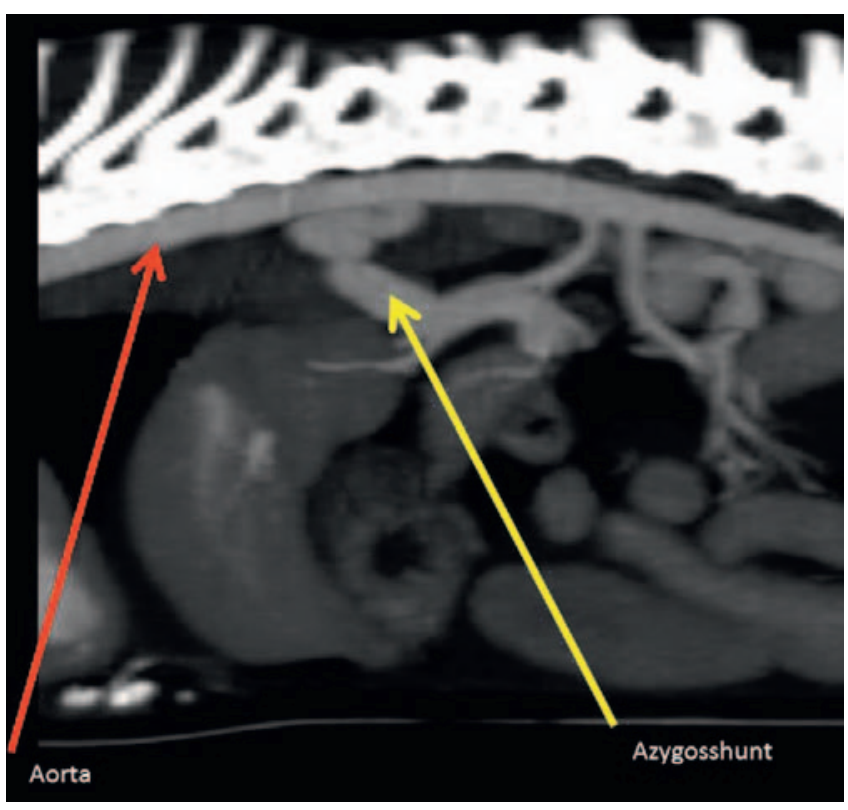

Abb. 8 Ein Portoazygosshunt im Maximum-Intensitätsprotokoll in eine lateralen Rekonstruktion (Schichtdicke $4 \mathrm{~mm}$ ). Die typische Schleife des Azygosshunts lässt sich in dieser Art der Darstellung sehr gut nachvollziehen.

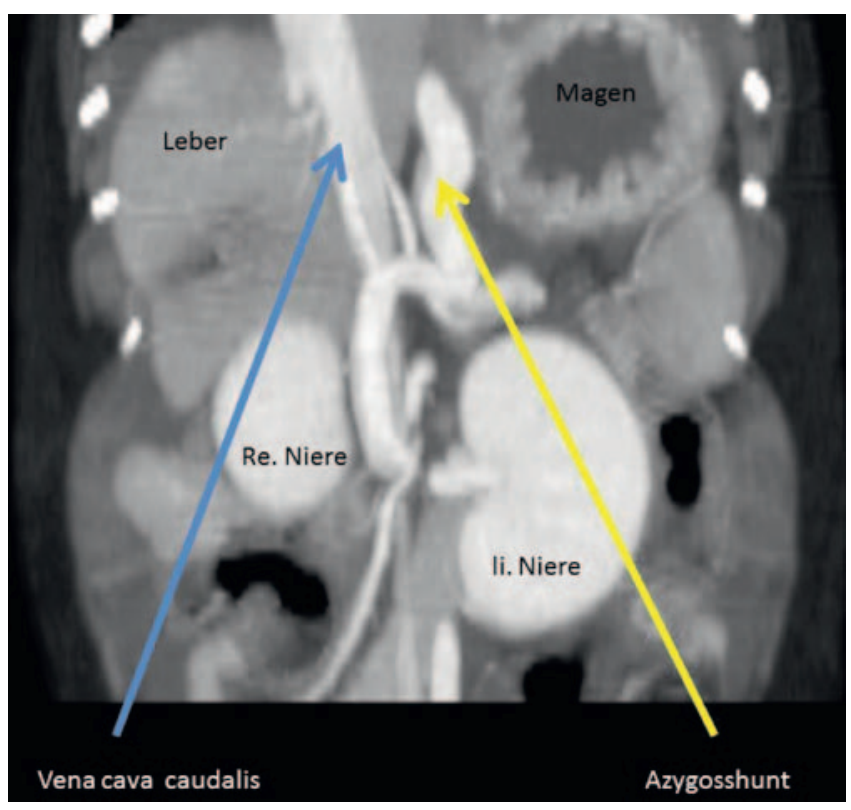

Abb. 9 Identischer Patient in einer koronaren Darstellung, jedoch etwas weiter ventral, weshalb nun die Aorta nicht mehr im Bild ist, jedoch auch die V. cava caudalis und beide Nieren mit Kontrastmittel angereichert sind. 


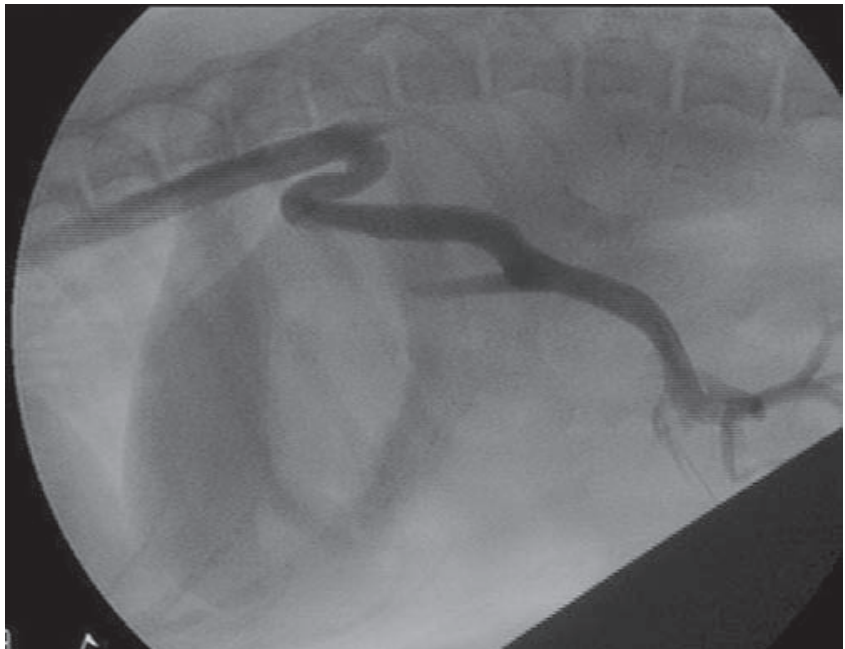

Abb. 10 Portoazygosshunt nach Applikation über eine Jejunalvene. Auch hier zeigt sich die typische Schleife des Shunts. Mit der jejunalen Portografie wird in der Regel das Portogramm am kontrastreichsten dargestellt.

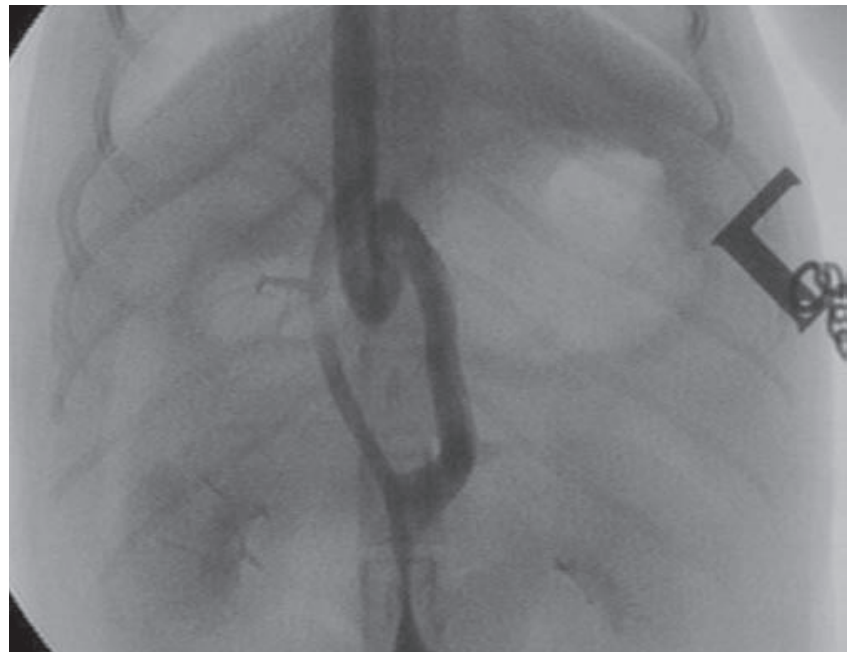

Abb. 11 Gleicher Shunttyp im dorsoventralen Strahlengang. Auch hier kann die für diese Shuntart typische Schleife erkannt werden. auswertbare Bilder. Die Anflutung des Kontrastmittels ist von Parametern wie Patientengröße, Herzfrequenz usw. abhängig, sodass diese zur Ermittlung des richtigen Untersuchungszeitpunkts in die Berechnung miteinbezogen werden sollten. Im Allgemeinen funktioniert diese Methode sehr gut, bei einzelnen Ausreißern kommt es aber dazu, dass der richtige Untersuchungszeitpunkt verpasst wird [3].

Alternativ dazu wird ein kleiner Kontrastmittel-Testbolus appliziert und gemessen, wie lange der Testbolus braucht, bis er in der V. portae maximal anflutet $[31,32,33]$. Anschließend wird dann der eigentliche Untersuchungsbolus injiziert und die Untersuchung mit der gemessenen Verzögerung gestartet.
Die zurzeit modernste Methode ist das Bolustracking [3]. Bei diesem Verfahren wird eine Region of Interest (ROI) in der V. portae positioniert und eine Schwelle definiert, meist 100 Hounsfield-Einheiten. Nach Applikation des Kontrastmittels misst der Tomograf sekündlich den Wert in der ROI und löst die eigentliche Untersuchung nach Erreichen des Schwellenwerts aus. Dies führt dazu, dass man den Zeitpunkt der optimalen Kontrastierung der V. portae sicher trifft. Durch den Einsatz weiterer Bildverarbeitungstechniken kann dann der Shuntverlauf noch deutlicher und sogar in 3D in allen Ebenen dargestellt werden.

Es muss kritisch darüber nachgedacht werden, inwieweit das Verfahren sinnvoll eingesetzt wird, insbesondere, weil

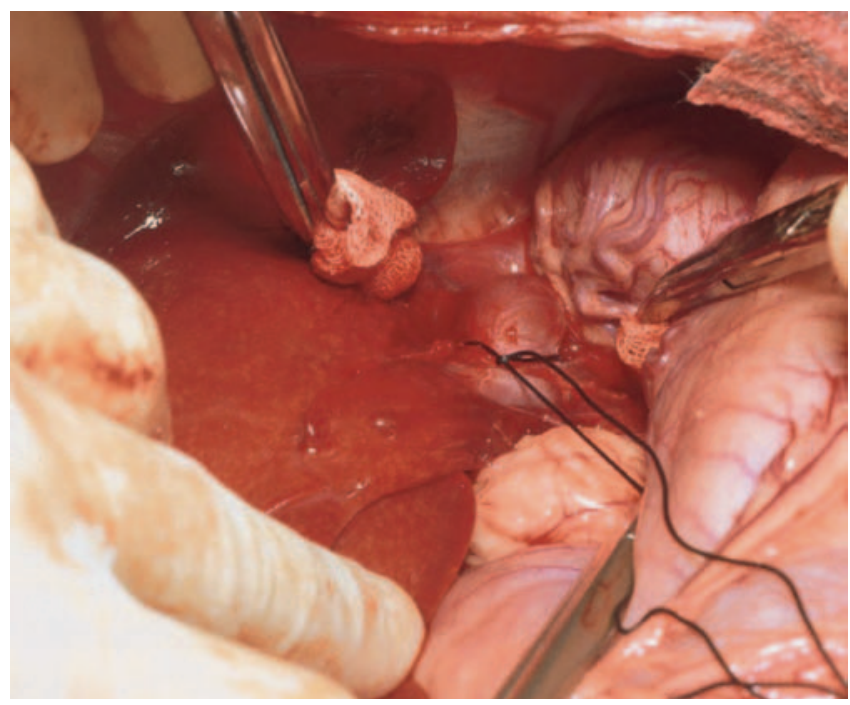

Abb. 12

Blick auf den OP-Situs eines portocavalen Shunts. Der Faden liegt bereits um das Shuntgefäß und engt dieses partiell ein. In einer 2. Operation wurde dann das Gefäß vollständig verschlossen. auf den Besitzer erhebliche Kosten zukommen. Die Vorteile des Verfahrens liegen jedoch auf der Hand: Es kostet wenig Zeit und man erhält nicht invasiv eine sehr genaue Vorstellung über Größe und Verlauf des Shunts. Mit diesen Informationen kann der Chirurg mit dem Besitzer noch einmal über Prognose und Risiko sprechen. Dieser hat dann die Möglichkeit, zum Beispiel bei arteriovenösen Fisteln oder komplizierten intrahepatischen Shunts, auf die Operation zu verzichten oder auf eine andere Therapieform umzuschwenken. Die CT gilt in der Shuntdiagnostik als sehr sicher, vorausgesetzt die Diagnostik wird technisch korrekt durchgeführt.

\section{Jejunale Portografie}

Die jejunale Portografie gilt nach wie vor als die exakteste Methode zur Diagnostik des PSS [14, 30]. Nach dem Eröffnen des Abdomens wird eine Jejunalschlinge vorgelagert und in eine Jejunalvene ein venöser Zugang gelegt. Unter Durchleuchtungskontrolle wird anschließend das Kontrastmittel appliziert und so der Shuntverlauf dargestellt ( Abb.10, - Abb. 11.).

Die Diagnostik kann direkt zur Therapie eingesetzt werden und gegebenenfalls die Therapie (Einengung des Gefäßes) auch direkt kontrolliert werden ( Abb. 12). Sollte das Tier jedoch keinen PSS haben, ist der Aufwand für das Tier unverhältnismäßig groß. 


\section{Zusammenfassung}

Zusammenfassend bietet einzig die Bildgebung einen sicheren Nachweis, ob ein PSS vorliegt. Solange nicht durch eine Portografie bewiesen ist, dass der Patient keinen Shunt hat, sollte diese Differenzialdiagnose nach wie vor als möglich angesehen werden.

Welche Methode gewählt wird, hängt letztlich von den Ergebnissen der Voruntersuchungen ab. Sonografisch ist der positive Nachweis des Shunts beweisend, der negative jedoch überhaupt nicht ausschließend. Geht es darum, bei einem Patienten einen durch die Voruntersuchungen unwahrscheinlichen Shunt sicher auszuschließen, erscheinen Splenoportografie und CT sinnvoll. Geht es darum, sich einen genauen Überblick (so nicht vorher möglich) über die Art des PSS zu machen, erscheint die CT die Modalität der Wahl. Wenn Patienten aufgrund der Klinik und Voruntersuchungen (z.B. sonografischer Nachweis) sehr sicher einen Shunt haben und dieser auch sicher operiert werden soll, so führt kein Weg an der jejunalen Portografie vorbei.

Alle Abbildungen @ Ingmar Kiefer

\section{Online zu finden unter}

http://dx.doi.org/10.1055/s-0033-1361513

\section{Literatur}

1 Bahr A. Der portosystemische Shunt beim Hund [Dissertation]. Berlin: Freie Universität Berlin Fachbereich Veterinärmedizin; 2006

2 Bernhardt N, Westhoff A, Meyer-Lindenberg A et al. Scintigraphic study for the diagnosis of portosystemic shunts in dogs. Dtsch Tierarztl Wochenschr 1996; 103: 183-186

3 Bosch B. Multiphasen Computertomografie der Leber beim Hund [Dissertation]. Leipzig: Veterinärmedizinische Fakultät; 2010

4 Bosch B, Kiefer I, Oechtering G et al. Computertomografie - häufig unterschätzt, selten überschätzt. kleintier konkret 2008; 3: 28-34

5 Bostwick DR, Twedt DC. Intrahepatic and extrahepatic portal venous anomalies in dogs: 52 cases (1982-1992). J Am Vet Med Assoc 1995; 206: 1181-1185

6 Bridger N, Glanemann B, Neiger R. Comparison of postprandial and ceruletide serum bile acid stimulation in dogs. J Vet Intern Med 2008; 22: 873-878

7 Center SA, Magne ML. Historical, physical examination, and clinicopathologic features of portosystemic vascular anomalies in the dog and cat. Semin Vet Med Surg (Small Anim) 1990; 5: 83-93

8 Daniel GB, Bright R, Ollis P et al. Per rectal portal scintigraphy using 99 mtechnetium pertechnetate to diagnose portosystemic shunts in dogs and cats. J Vet Intern Med 1991; 5: 23-27

9 D’Anjou MA, Penninck D, Cornejo L et al. Ultrasonographic diagnosis of portosystemic shunting in dogs and cats. Vet Radiol Ultrasound 2004; 45: 424-437

10 Faverzani S, Trombetta R, Grieco V et al. Clinical, laboratory, ultrasonographic and histopathological findings in dogs affected by portosystemic shunts, following surgery or medical treatment. Vet Res Commun 2003; 27: 755-758

11 Fossum TW. Portosystemic vascular anomalies. In: Fossum TW, Hrsg. Small Animal Surgery. 2. Aufl. St. Louis: Mosby-Year Book; 2002: 457-46810

12 Frank P, Mahaffey M, Egger C et al. Helical computed tomographic portography in ten normal dogs and ten dogs with a portosystemic shunt. Vet Radiol Ultrasound 2003; 44: 392-400

13 Gerritzen-Bruning MJ, van den Ingh TS, Rothuizen J. Diagnostic value of fasting plasma ammonia and bile acid concentrations in the identification of portosystemic shunting in dogs. J Vet Intern Med 2006; 20: 13-19

14 Grevel V, Schmidt S, Lettow E et al. Der angeborene portosystemische Shunt bei Hund und Katze. Tierarztl Praxis 1987; 15: 77-92; 185-194

15 Hiller A. Der kongenitale portosystemische Shunt beim Hund: retrospektive Untersuchung zur Prävalenz, den Risikofaktoren und der Genetik [Dissertation]. Hannover: Tierärztliche Hochschule; 2009

16 Holt DE, Schelling CG, Saunders HM et al. Correlation of ultrasonographic findings with surgical, portographic, and necropsy findings in dogs and cats with portosystemic shunts: 63 cases (1987-1993). J Am Vet Med Assoc 1995; 207: 1190-1193

17 Hunt GB. Effect of breed on anatomy of portosystemic shunts resulting from congenital diseases in dogs and cats: a review of 242 cases. Aust Vet J 2004; 82: 746-749

18 Kerr MG, van Doorn T. Mass screening of Irish wolfhound puppies for portosystemic shunts by the dynamic bile acid test. Vet Rec 1999; 144: 693-696

19 Krotschek U, Adin CA, Hunt GB et al. Epidemiolocic factors associated with the anatomic location of intrahepatic portosystemic shunts in dogs. Vet Surg 2007; 36: 31-36

20 Lamb CR. Ultrasonographic diagnosis of congenital portosystemic shunts in dogs: result of a prospective study. Vet Radiol Ultrasound 1996; 37: 281-288
21 Maddison JE. Canine congenital portosystemic encephalopathy. Aust Vet J 1988; 65: 245-249

22 Meyer HP, Rothuizen J. Congenital portosystemic shunts (PSS) in dogs are a genetic disorder. Tijdschr Diergeneeskd 1991; 116: $80 S-815$

23 Meyer HP, Rothuizen J, Ubbink G] et al. Increasing incidence of hereditary intrahepatic portosystemic shunts in Irish wolfhounds in The Netherlands (1984 to 1992). Vet Rec 1995; 136: 13-16

24 Meyer HP, Rothuizen J, Tiemessen I et al. Transient metabolic hyperammonaemia in young Irish wolfhounds. Vet Rec 1996; 138: 105-107

25 Tisdall PLC, Hunt GB, Bellenger CR et al. Congenital portosystemic shunts in Maltese and Australian cattle dogs. Aust Vet J 1994; 71: 174-178

26 Tobias KM. Determination of inheritance of single congenital portosystemic shunts in Yorkshire Terriers. J Am Anim Hosp Assoc 2003; 39: 385-389

27 Tobias KM, Rohrbach BW. Association of breed with the diagnosis of congenital portosystemic shunts in dogs: 2400 cases (1980-2002). J Am Vet Med Assoc 2003; 223: 1636-1639

28 Ubbink G], van de Broek J, Meyer HP et al. Prediction of inherited portosystemic shunts in Irish wolfhounds on the basis of pedigree analysis. Am J Vet Res 1998; 59: 1553-1556

29 van Straten G, Leegwater PA, de Vries M et al. Inherited congenital extrahepatic portosystemic shunts in Cairn terriers. J Vet Intern Med 2005; 19: 321-324

30 White RN, MacDonald NJ, Burton CA. Use of intraoperative mesenteric portovenography in congenital portosystemic shunt surgery. Vet Radiol Ultrasound 2003; 44: 514-521

31 Zwingenberger AL, Schwarz T. Dual-phase CT angiography of the normal canine portal and hepatic vasculature. Vet Radiol Ultrasound 2004; 45 (2): 117-124

32 Zwingenberger AL, Schwarz T, Saunders HM. Helical computed tomographic angiography of canine portosystemic shunts. Vet Radiol Ultrasound 2005; 46 (1): 27-32

33 Zwingenberger AL, Shofer FS. Dynamic computed tomographic quantitation of hepatic perfusion in dogs with and without portal vascular anomalies. Am J Vet Res 2007; 68 (9): 970-974

\section{Dr. med. vet. Ingmar Kiefer \\ Dr. med. vet. Beate Bosch \\ TÄ Claudia Köhler \\ TÄ Franziska Eberhardt \\ PD Dr. Eberhard Ludewig \\ Klinik für Kleintiere \\ Universität Leipzig}

An den Tierkliniken 23, 04103 Leipzig 


\section{Fragebogen}

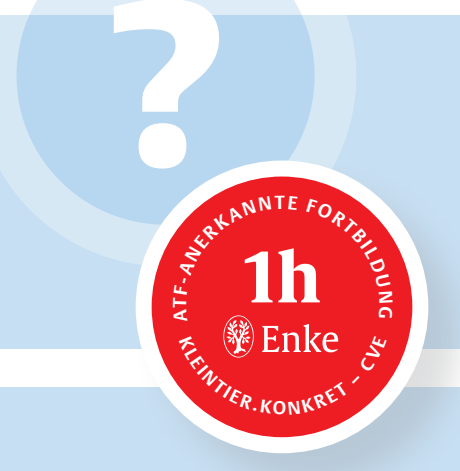

\section{Frage 1}

Bei welcher Rasse empfiehlt der Zuchtverband routinemäßig eine Bestimmung des Ammoniakwerts bei den Jungtieren?
a. Yorkshire Terrier
b. Golden Retriever
c. Malteser
d. Irischer Wolfshund
e. Cairn Terrier

\section{Frage 2}

Von welchem Verhältnis in Bezug auf das Vorkommen von extra- und intrahepatischem Shunt wird im Allgemeinen ausgegangen?
a. $1: 1$
b. $2: 1$
c. $3: 1$
d. $1: 2$
e. $1: 3$

\section{Frage 3}

Der Anteil der portalen Durchblutung zum gesamten Blutfluss in der Leber liegt beim gesunden Hund bei ca. wie viel Prozent?
a. $30 \%$
b. $40 \%$
c. $50 \%$
d. $70 \%$
e. $90 \%$

\section{Frage 4}

Wodurch fallen Ammoniumurate bei der sonografischen Untersuchung der Blase auf?
a. nicht darstellbar
b. schnelle Sedimentation
c. zeigen keinen distalen Schallschatten
d. Art Schwebeverhalten
e. Klumpenbildung

\section{Frage 5}

Welcher Labortest zum Shuntnachweis wird aufgrund seiner Unverträglichkeit bei Shuntpatienten kaum noch eingesetzt?
a. Gallensäurenstimulation mit Ceruletid
b. Gallensäurenstimulation mit Reiz- mahlzeit
c. Ammoniaktoleranztest
d. Cholesterinbestimmung
e. Fütterungstest

\section{Frage 6}

Welche bildgebende Methode wird als „Goldstandard“ in der Shuntdiagnostik angesehen?
a. jejunale Portografie
b. Splenoportografie
c. rektale Szintigrafie
d. Sonografie
e. Projektionsradiografie

\section{Frage 7}

Mit welchem CT-Verfahren ist es am sichersten möglich, den korrekten Untersuchungszeitpunkt zur Darstellung der Portaldurchblutung zu ermitteln?
a. Schätzung
b. Berechnung anhand einfacher klinischer Parameter
c. Test-Bolus
d. Bolustracking
e. anhand der Standardwerte

\section{Frage 8}

Welche Shuntart tritt beim Irischen Wolfshund am häufigsten auf?
a. intrahepatischer Shunt
b. erworbener Shunt
c. Azygosshunt
d. keine Aussage möglich
e. Phrenicoshunt

\section{Frage 9}

In welchem Teil des Gastrointestinaltrakts entsteht das Ammoniak?
a. Magen
b. Duodenum
c. Jejunum
d. Kolon
e. Ileum

\section{Frage 10}

Wie stellen sich die Nieren von Shuntpatienten meist radiologisch dar?
a. zu klein
b. zu groß
c. normal groß
d. keine Aussage möglich
e. eine Niere verkleinert, die zweite dadurch reaktiv vergrößert




\section{Klinisches Bild und Diagnostik des angeborenen portosystemischen Shunts beim Hund}

Ingmar Kiefer, Beate Bosch, Claudia Köhler, Franziska Eberhardt, Eberhard Ludewig

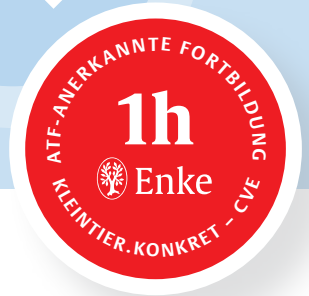

A Lernerfolgskontrolle

\section{Bitte kreuzen Sie die richtigen Antworten an! Es ist jeweils nur 1 Antwort pro Frage richtig!}

\section{B Teilnehmer}

Titel | Name | Vorname

Beruf

\section{Ihr Ergebnis wird vom Verlag ausgefüllt}

Sie haben_von Frage

richtig beantwortet und somit

Stuttgart, den

D Teilnahmebedingungen für Abonnenten der kleintier konkret kostenlos

Für diese Fortbildungseinheit können Sie 1 ATFFortbildungsstunde anerkannt bekommen. Hierfür

- müssen mindestens $70 \%$ der Fragen richtig beantwortet sein.

- muss der Antwortbogen vollständig ausgefüllt sein. Unvollständig ausgefüllte Bögen können nicht berücksichtigt werden!

\section{E Erklärung}

Ich versichere, dass ich die Beantwortung der Fragen selbst und ohne Hilfe durchgeführt habe.
Straße | Hausnummer

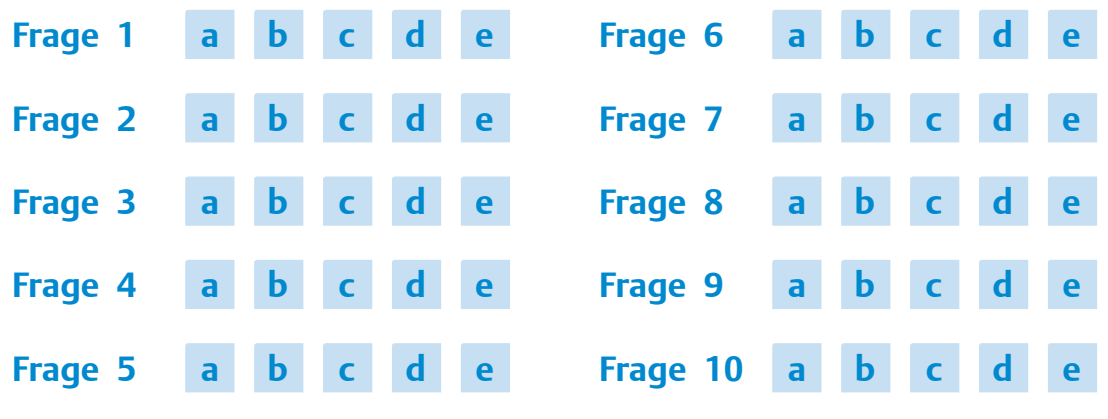

PLZ | Ort kleintier konkret-CVE-Wertmarken für NichtAbonnenten können beim Verlag zu folgenden Bedingungen erworben werden: 6erPackWertmarken, Preis 49,95€ inkl. MWSt., Artikel-Nr. 903000.

Bitte richten Sie die Bestellungen an: MVS Medizinverlage Stuttgart,

KundenServiceCenter Buch, Postfach 3011 20, 70451 Stuttgart.

${ }^{*}$ Nicht-Abonnenten bitte hier kleintier konkret-CVE-Wertmarke aufkleben, Abonnenten bitte Abonnentennummer eintragen 